\title{
Seasonal Agricultural Products Distribution and Traceability System Based on the RFID Internet of Things
}

\author{
Zhang Hongbo ${ }^{1}$ and Gao $\mathrm{Wei}^{1}$ \\ ${ }^{1}$ Department of Computer Science, Tangshan Normal College,Tangshan City, \\ Hebei Province, China \\ 'zhangbobo_728@sina.com.cn
}

\begin{abstract}
Based on the big data and complexity in the identification process of IOT radio frequency, this article puts forward an optimal algorithm for Adaptive Gausian Traversal Harmony Search IOT radio frequency identification, which aim at solving the problems of high computational complexity and low identification accuracy of traditional algorithm. At first, the network optimization design is carried out bolsed on Harmony Search (HS) algorithm, and in order to solve the problems of standard HS in optimization accuracy, computational complexity and other aspects, control parameters are introduced into the algorithm improvisation process on the basis of traverse characteristics of Gaussian function, so that the pertinence of preliminary and later searches are improved, and meanwhile, theoretical analysis on paraneters selection is described; Secondly, the optimization model of IOT radio frequency identification is studied and improved adaptive optimization objectives are put forward to achieve equilibrium optimization of performance indicators; at last, tes results are compared to prove the suggested algorithm having high computational accuracy and fuster computational speed.
\end{abstract}

Keywords: IOT; Radio frequency identiflcation; Logistics; Gaussian traverse; Harmony search; Self-adoptation

\section{Introduction}

With the rapid development of IOT (Internet of Things) technology, RFID (Radio frequency ident fication netyork) based IOT logistics management has become more and more popular. The RFIP system is mainly composed of three parts: Reader module, data management module and electronic tags; in the network, electronic tags and reader module are usually used together. Among them, the rationality of reader module configuration plays a key role in lowering network costs and reducing the configuration conflicts between electronic tag resources and reader module, and it is the focus and crucial topic for the research on radio frequency network optimization in IOT technology. This arficle mainly studies the RFID network optimization problems by using the HS algofithn, and meanwhile, it considers the optimization of objective function of logistic RHID network by using modified adaptive weighting coefficients. Harmony search algorithm (HS) is an intelligent optimization algorithm which was put forward by Geem and others in 2001 [8], and compared with conventional intelligent optimization algorithm, HS algorithm has the following advantages [9]: (a) In case of generating new harmony, HS algorithm will consider all existing harmonies while Particle Swarm Optimization and other algorithm only consider parent individuals; (b) Hs algorithm adjust the generating method of new harmony according to independent variables; (c) During the improvisation process of HS algorithm, the memory bank can be designed by using random numbers, which in nature is a kind of global random search process.

Based on the advantages of above-mentioned algorithm, this article considers using HS algorithm as basic optimization algorithm, and based on the characteristics of HS 
algorithm, it improves the improvisation process by using Gaussian Traverse Function, so as to enhance the pertinence of HS algorithm on real-time information in preliminary and later search, based on which corresponding adjustment will be made on the tracking and search strategies of real-time change of IOT RFID network; meanwhile, regarding the non-adaptability of IOT RFID network model, the article considers using adaptive weights to realize balanced design of IOT RFID network performance indicators.

\section{Gaussian Traverse Harmony Search}

\subsection{Harmony Search}

The optimization process of Harmony Search and music production process have many similarities. A couple of music creation concepts are introduced into HS algorithm, mainly including the following algorithm parameters: Harmony memory (HM) and its, size (HMS) and harmonic memory reference ratio (HMCR), etc., which are used for storing input vectors, characterizing vector sizes and current music parameter reference rate, $H M C R \in[0,1]$. In addition, there are other parameters, such as pitch adjustment ratio (PAR) and distance width adjustment ratio $(b w)$, etc. Detailed procedures of standard harmony search algorithm are as follows [10]:

Step1: (algorithm initialization) input vectors are stored in HM at first, and meanwhile, value range of input vectors are specified. Swarm individuals with a size of HMS are generated based on the following Formula (1), and are stored in HM:

$x_{i}^{r}=x_{i}^{l}+\operatorname{rand} \times\left(x_{i}^{u}-x_{i}^{l}\right)$

In Formula (1), and are the upper and lower linit values of Vector No. in vector individuals.

Step 2: (improvisation process) Re vectors are created by means of improvisation: (a) to select an existing vector in HM or to create a new vector based on HMCR is determined by random. (b) if it is deternimed to select an existing vector, the adjustment will be made on the basis of two parameters, PAR and $b w$, and see Formula (2) for detailed procedures:

$$
\begin{cases}x_{i}^{n e w}=x_{i}^{r} \pm \text { rand } \times b, & \text { if rand }<\mathrm{PAR} \\ x_{i}^{n e w}=x_{i}^{r}, & \text { if rand } \angle \mathrm{PAR}\end{cases}
$$

Step 3: (HM update process) To compute the adaptive value of new individual, and compare the value with that of original vector individual, then select the superior individual to partcipate next generation of evolution according to one-to-one elimination mechanism, so that the HM update process is completed.

Step 4: (algorithm stop discrimination) To check whether the value of existing optimal individual in current memory meet the requirement of algorithm termination, and if it is met, the discrimination is terminated and the superior individual will be output; otherwise, in willump to Step 2 to continue the improvisation process of next generation.

\subsection{Description of Refinement Algorithm}

In the improvisation process of HS algorithm, if the conditions of HMCR are met, existing vector individuals in HM will be selected. Then the adjustment will be made based on parameters PAR and $b w$. Among them, the selection of parameter $b w$ has a profound impact on the performance of $\mathrm{HS}$ algorithm, with larger $b w$ values going against further development of the algorithm, smaller values going against the extent exploration of the algorithm, and fixed $b w$ values unable to make automatic adjustment according to the evolution conditions of the algorithm; the above-mentioned disadvantages are obvious and major disadvantages of HS algorithm. The reasonable strategy is selecting larger $b w$ 
values in preliminary period and smaller $b w$ values in later period, which can improve the algorithm performance [11]. Control parameter ${ }^{\alpha}$ can be used:

$\alpha=1-\lambda \times$ iter $_{\text {current }} /$ iter $_{\max }$

At the same time, Gaussian ergodicity can be used for the refinement of improvisation, and it can be used together with the iteration by taking larger probability values and smaller step iteration to further develop existing positions, and by taking larger probability values and larger step iteration to explore the extent of future positions; and based on control parameter ${ }^{\alpha}$ and Gaussian traverse function ${ }^{g}$, the refinement of creation process is realized:

$\begin{cases}x_{i}^{\text {new }}=x_{i}^{r} \pm g \times a \times b w, & \text { if } \text { rand }<\mathrm{PAR} \\ x_{i}^{\text {new }}=x_{i}^{r}, & \text { if rand } \geq \mathrm{PAR}\end{cases}$

In Formula (4), $g \sim \mathrm{N}\left(\mu, \sigma^{2}\right)$, the distribution (variance) $\sigma$ of Gaussian traverse function $g$ has a significant impact on the performance of HS algorithm.

\subsection{Theoretical Analysis}

In order to facilitate the theory analysis of the algonthm, it is assumed that vector individuals stored in HM are all in one dimensional distribution, Mith the following forms:

$\mathrm{HM}=x=\left\{x_{1}, \cdots, x_{\mathrm{HMS}}\right\}^{T}$

The adaptive values, to which vector individuals orrespond, are all one dimensional vectors, with the following forms:

$y=\left\{y_{1}, \cdots, y_{\text {нмS }}\right\}^{T}$

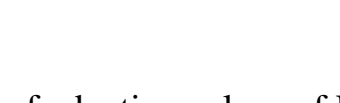

The distribution variance of adaptive values of $\mathrm{HS}$ algorithm evolution swarm is:

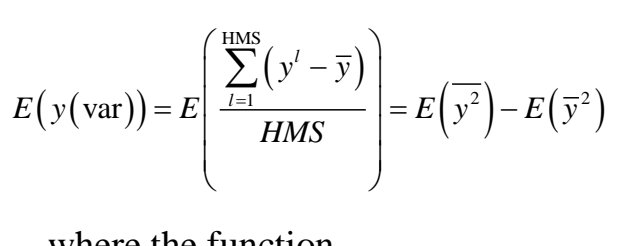

where the function,

$E\left(\overline{y^{2}}\right)=\mathrm{HMCR} \cdot \overline{x^{2}}+\mathrm{HMCR} \cdot \mathrm{PAR} / 3$

$$
+(1-\mathrm{HMCR})\left(a^{2}+a b+b^{2}\right) / 3
$$

$E\left(\bar{y}^{2}\right)\left(\left(y^{i}\right)^{2}+\frac{(\mathrm{HMS}-1) \cdot\left(E\left(y^{i}\right)\right)}{\mathrm{HMS}}\right)^{2}$

then, the distribution variance of one dimensional adaptive values is:

$$
\begin{aligned}
E(y(\mathrm{var})) & =\left(1-\frac{1}{\mathrm{HMS}} \cdot \mathrm{HMCR} \cdot E(x(\mathrm{var}))\right) \\
& +\mathrm{HMCR} \cdot(1-\mathrm{HMCR}) \cdot \bar{x}^{2}+\frac{b \omega^{2}}{3} . \\
& \mathrm{HMCR} \cdot \mathrm{PAR}+\frac{(1-\mathrm{HMCR})}{12}(a-b)^{2}
\end{aligned}
$$


In Formula (10), $[a, b]$ is the value range of one dimensional individual $x$, and $y$ (var) and $y$ (var) correspond to the distribution variance of individual $x$ and its adaptive value $y$, respectively. When $E(y($ var $))$ is too large, HS algorithm focuses on exploration, but it is easy to cause algorithm divergence. When $1 / \mathrm{HMS}$ is too small and HMCR $\rightarrow 1$, Formula (10) can be reduced to:

$E(y(\mathrm{var}))=\operatorname{HMCR}\left(E(x(\mathrm{var}))+\frac{b \omega^{2} \cdot \mathrm{PAR}}{3}\right)$

According to the HS algorithm improvisation process (4), Formula (11) can be rewritten as:

$$
E(y(\operatorname{var}))=\mathrm{HMCR} \cdot\left(E(x(\operatorname{var}))+\frac{b \omega_{0}^{2} \alpha^{2} g^{2} \cdot \mathrm{PAR}}{3}\right)
$$

In Formula (12), the values change along the decrease iteration of parameter $\alpha$ which is advantageous to convergence $E(y(\mathrm{var}))$ and can effectively prevent AS/gorithm divergence. After introducing Gaussian parameter $g$, the creation process complies with Gaussian traverse distribution, which can avoid monotonic decrease of $\alpha^{2} f^{2}$, invigorate the algorithm and effectively balance the exploration and development capabilities of the algorithm.

\section{RFID Optimization of GTHS Logistics}

\subsection{Description of RFID Model}

RFID logistics optimization proces is essentially a kind of target optimization process; and according to the above descriptions of harmony search algorithm, before using it for the optimization of logistics RFID network the target function model should be established, and the reasonableness of the model has certain impact on optimization result. Similar to the structure form of target function of common optimization algorithm, Reference [12] makes a profound research on logistics RFID problem and designs an optimization computation targe/model, but Reference [12] uses the target function with fixed weighting farameter, whosedetailed form is:

$$
\min f=\gamma_{1} f_{1}+\ldots+\gamma_{n} f_{n}
$$

In Formula (13), is the weighting factor (fixed weight) of each performance index, and $f_{i}$ is the performance index which meets:

$$
\sum_{i=1}^{n} \gamma_{i}=1
$$

It is assumed that in IOT RFID network area, the ceiling number of readers is $N_{\max }$, and the actual number of readers is $N_{R}$, and the number of electronic tags is $N_{t}$, the signal coverage radius of reader module is $R_{R}$, and the position is $\left(x_{S}^{i}, y_{S}^{i}\right)$; if the network space is $A$, the optimization feasible region of IOT RFID problem is:

$\left(x_{S}^{i}, y_{S}^{i}\right) \in A, \forall i \in\left\{1, \cdots, N_{R}\right\}$

If electronic tag $j$ is located at $\left(x_{o}^{i}, y_{o}^{i}\right)$, it can be defined that:

$\left\{\begin{array}{l}S N_{R}=\left\{1, \cdots, N_{R}\right\} \\ S N_{t}=\left\{1, \cdots, N_{t}\right\}\end{array}\right.$ 
Then the distance between reader $i$ and electronic tag $j$ in IOT RFID network can be computed by using the formula:

$d\left(s_{i}, o_{j}\right)=\sqrt{\left(x_{S}^{i}-x_{o}^{i}\right)^{2}+\left(y_{S}^{i}-y_{o}^{i}\right)^{2}}$

\subsection{Definition of Impact Factor}

3.2.1. RFID Network Coverage: If the value range of electronic tags in RFID network is $R_{T}$, the identification capability of reader ${ }^{i}$ on electronic tag ${ }^{j}$ is:

$r d e r_{i, j}=\left\{\begin{array}{l}0, R_{R}+R_{T} \leq d\left(s_{i}, o_{j}\right) \\ 1, R_{R}-R_{T}>d\left(s_{i}, o_{j}\right) \\ e^{\left(-\lambda_{1} \alpha_{1} \beta_{1}^{\beta} \alpha_{2} \beta_{2}+\lambda_{2}\right)}, \text { otherwise }\end{array}\right.$

In Formula (18), the parameters can be defined as: $\alpha_{1}=R_{T} R_{R} d\left(s_{i}, o_{j}\right)$, $\alpha_{2}=R_{T}+R_{R}-d\left(s_{i}, o_{j}\right)$, where, $\lambda_{1}$ is called perception parameter and $\lambda_{2}$ is called noise parameter. Then, the formula of IOT RFID network coverage is

$c_{O}^{j}=1-\prod_{i \in S N_{R}}\left(1-r d e r_{i, j}\right)$

The network coverage index can be defined as.

$f_{1}=\frac{100}{N_{t}} \sum_{j \in S N_{t}} c_{o}^{j}$

In order to express it in the formof minimum index, the following formula can be used to make refinement:

$f_{1}=100-\frac{100}{N_{t}} \sum_{j \in S N_{t}} c_{O}^{j}$

3.2.2. RFID Network Operation Cost: The ceiling number of network readers is $N_{\max }$ and the actual number of readem module in the network is $N_{R}$, then cost of readers in RFID network is:

$f_{2}=\frac{N_{\max }-N_{R}}{N_{\max }}$

3.2.3. RFID Network Load Balancing: It is very difficult to establish the load balancing mathematicál model, but an approximate model can be established by using Monte Carlo sampling. It is assumed that the electronic tag $j$ is located at $o_{k}^{j}\left(x_{k}^{\prime j}, y_{k}^{\prime j}\right)$, in which the sampling point No. ${ }^{k}$ corresponds the reader ${ }^{i}$, then it can be obtained by Formula (18) that $d e r_{i, j}^{j}=1$, otherwise ${ }^{r d e r_{i, j}^{j}}=0$, and it can be obtained:

$r d e r_{i, j}^{j}=\left\{\begin{array}{l}0, R_{R}+R_{T}<d\left(s_{i}, o_{j}\right) \\ 1, R_{R}-R_{T} \geq d\left(s_{i}, o_{j}\right)\end{array}\right.$

In Formula (23), $k=1, \cdots, K, K$ is the Monte Carlo sampling point, and then the following conditions are met:

$R_{R}-R_{T}<d\left(s_{i}, o_{j}\right) \leq R_{R}+R_{T}$ 
The polar coordinate form of sampling points can be obtained based on the method of concentric circles:

$$
(r, \theta)=\left\{\begin{array}{l}
(0,0), \text { if } \varepsilon_{1}=\varepsilon_{2}=0 ;\left(\varepsilon_{1}, \frac{\pi \varepsilon_{2}}{4 \varepsilon_{1}}\right), \text { if } \varepsilon_{1}>\left|\varepsilon_{2}\right| ; \\
\left(\varepsilon_{2}, \frac{\pi\left(2-\varepsilon_{1} / \varepsilon_{2}\right)}{4}\right), \text { if }-\varepsilon_{2}<\varepsilon_{1} \leq \varepsilon_{2} ; \\
\left(-\varepsilon_{1}, \frac{\pi\left(4+\varepsilon_{2} / \varepsilon_{1}\right)}{4}\right), \text { if } \varepsilon_{1} \leq-\varepsilon_{2} \cup \varepsilon_{1}<\varepsilon_{2} ; \\
\left(-\varepsilon_{2}, \frac{\pi\left(6-\varepsilon_{1} / \varepsilon_{2}\right)}{4}\right), \text { otherwise. }
\end{array}\right.
$$
is:

Then, the overlapping degree of sampling point $k$ within the range of electronic tag $j$

$$
\operatorname{oer}_{k}=\left\{\begin{array}{l}
0, \sum_{i \in S N_{R}} r d e r_{i j}^{j} \leq 1 \\
\sum_{i \in S N_{R}} r d e r_{i j}^{j}-1, \sum_{i \in S N_{R}} r d e r_{i j}^{j} \geq 2
\end{array}\right.
$$

and load balancing index can be defined as:

$$
f_{3}=\prod_{j \in S N_{t}} \frac{1}{1+\frac{1}{k} \sum_{i \in S K} o e r_{k}^{j}}
$$

\subsection{Adaptive Target Function}

In order to solve the integration problem of evaluation indexes of the above-mentioned three IOT RFID network performance, the traditional solution is to comprehensively consider the three indexes by using weighting factor, and establish the comprehensive target function of RFID network, with the cetailed form as follows:

$$
\begin{aligned}
& \min f=\gamma_{1} f_{1}+\gamma_{2} f_{2}+\gamma_{3} f_{3} \\
& \text { In Formula (28), } \gamma_{1}, \gamma_{2}, \gamma_{3} \text { are yeightings, and shall meet following requirement: } \\
& \gamma_{1}+\gamma_{2}+\gamma_{3}=1
\end{aligned}
$$

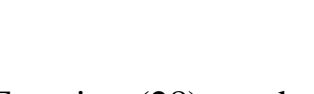

Target Function (28) can be divided into two situations: one is when $f_{1}, f_{2}$ and $f_{3}$ are in the same order of magnitude, $\gamma_{1}, \gamma_{2}, \gamma_{3}$ can use a same order of magnitude; the other is when $f_{1}, f_{2}$ and $f_{5}$ are in different orders of magnitude or one of them is in a different order of magnitude, then the values of $\gamma_{1}, \gamma_{2}, \gamma_{3}$ should be selected according to the orders of magnitude of $f_{1}, f_{2}$ and $f_{3}$. But in practical application, the orders of magnitude of $f_{1}$, $f_{2}$ and continuously vary with the performance index characteristics and evolution process, therefore, the target function with fixed weighting cannot reach ideal results. In order to solve this problem, an adaptive weighting selection method is designed as follows:

$\min f=\frac{f_{1}-f_{1}^{\text {min }}}{f_{1}^{\text {max }}-f_{1}^{\text {min }}}+\frac{f_{2}-f_{2}^{\text {min }}}{f_{2}^{\max }-f_{2}^{\text {min }}}+\frac{f_{3}-f_{3}^{\text {min }}}{f_{3}^{\max }-f_{3}^{\text {min }}}$

Where, $f_{i}^{\min }$ and $f_{i}^{\max }$ are the minimum values and maximum values of No. $i$ Index $f_{i}$ in current evolution swarm. In the above-mentioned adaptive target function, the hyperplane defined by index weighting and limiting points shall be updated along with the update of index limiting points, and the final result will be minimum limiting point 
gradually approaching final ideal point, so that the optimization of target function is completed.

\section{Experimental Analysis}

\subsection{Algorithm Parameters Impact}

Two standard test functions are selected for the performance test of GTHS algorithm, and the characteristics of the two functions include high dimensions, multi peaks, lots of local extreme points which are strongly seductive to HS individuals; the test functions are as shown in Formula (31), and see Table 1, for the overall optimal values and parameter settings.

$$
\begin{aligned}
& f_{1}(X)=\sum_{i=1}^{n}\left[x_{i}^{2}-10 \cos \left(2 \pi x_{i}\right)+10\right], \\
& f_{2}(X)=\left[\sum_{i=1}^{n} x_{i}^{2}-\prod_{i=1}^{n} \cos \left(x_{i} / \sqrt{i}\right)+1\right] / 4000 .
\end{aligned}
$$

Table 1. Test Function Parameter Settings

\begin{tabular}{cccc}
\hline $\mathrm{f}$ & $\mathrm{D}$ & $\mathrm{S}$ & $\mathrm{P}$ \\
\hline $\mathrm{f} 1$ & 20 & {$[-5.12,5.12]$} & 0 \\
$\mathrm{f} 2$ & 20 & {$[1-600,600]$} & 0 \\
\hline
\end{tabular}

In the above-mentioned refinement algorithm, the main way is to add Gaussian traverse parameter $g$ and control parameter $\alpha$ into HS algorimn, and $\lambda$ has a large impact on parameter $\alpha$, so does variance $\sigma_{g}$ on Gaussian trầerse parameter $g$. Parameter settings: $\mathrm{HMS}=100, \mathrm{HMCR}=0.95, \mathrm{PAR}=0 . \mathrm{D}_{\mathrm{te}}=1500, \lambda=0.3$. The test results are as shown in Figure 1 .

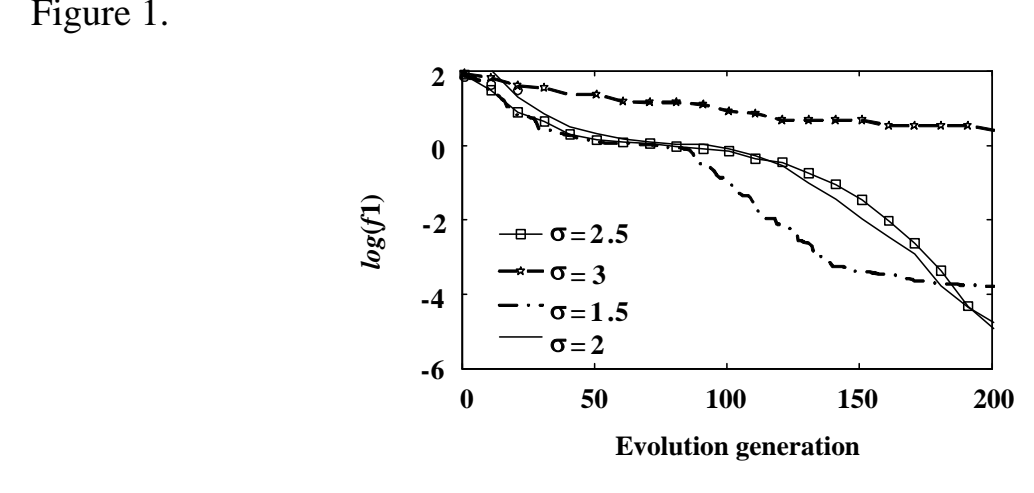

(a) f1 function

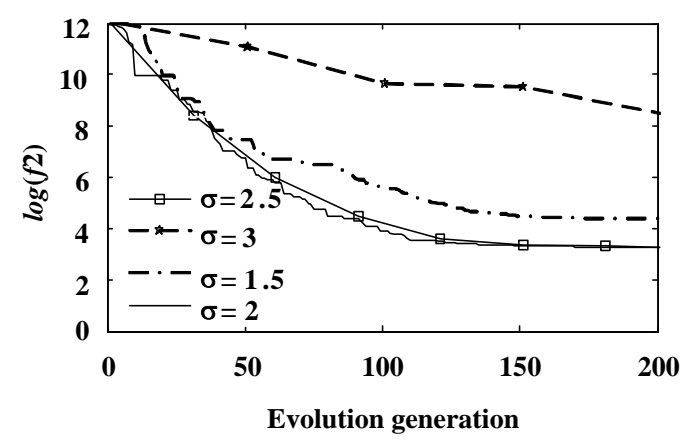

(b) f2 function 


\section{Figure 1. Effect of Variance Value}

It can be seen from the comparison of curves in Figure 1 (a), and (b), when variance $\sigma_{g}=2$, HS algorithm has the optimal convergence process. Meanwhile in theory, it can be seen from the analysis of Formula deviation (12), the main function of parameter $\alpha$ is to make the values of parameter $b w$ in a state of monotonic decreasing; larger $b w$ can be used in preliminary stage of the algorithm and smaller $b w$ can be used at later stage, which can balance the exploration and development capabilities in both preliminary and later stages of the algorithm. In spite of this, as the change of $b w$ is too regular, the innovation of this algorithm will be reduced. In this regard, the Gaussian traverse function $g$ is used to enhance the randomness of $b w$ change, but in fact, the value of $g$ is not as large as better, and it can be seen from the analysis of simulation comparing results, $\sigma_{g}=2$ is the optimal value.

As a matter of fact, like Figure 2, in terms of Gaussian traverse function $g \sim N(0, \sigma)$, if the Gaussian function variance $\sigma$ is too small (for example $\sigma=0.5$ ), the values of $y$ will be mainly distributed within $[-1,1]$, showing a peak pattern, and the overall distribution values are comparatively larger. While when variance $\sigma$ is too large (for example $\sigma=4$ ), the value distribution of $y$ will show a flat pattern, mainly within $[-6,6]$; and the transverse space is expanded while the overall values of $y$ are comparatively smaller. The specific impacts on control parameters: (a) large $y$ values will have larger impact on parameter $\alpha$, and meanwhile, it will disturb the overall decreasing trend of $b w$, (b) small $y$ values will have little impact on parameter $\alpha$, and cannot show the improvement impact of Gaussian traverse distribution $g$ on control parameter $\alpha$.

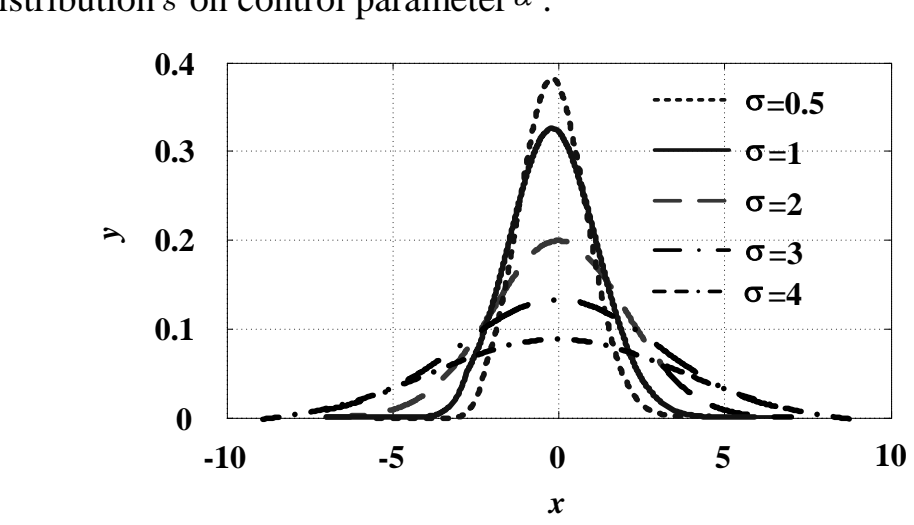

Figure 2. Gauss Distribution for Different $\sigma$

\subsection{Example of IOT RFID Optimization}

According to the experiment setting in Reference [9], the IOT RFID network area is $30 \mathrm{~m} \times 30 \mathrm{~m}$ and there are approximately 100 electronic tags randomly distributed in this area, and each reader has a deployment ceiling of $N_{\max }=20$; electronic tag setting area has a radius parameter uncertainty value of $R_{T}=1 \mathrm{~m}$, and the detection radius of the reader is $R_{R}=8 \mathrm{~m}$; the parameter value of RFID network coverage index is:

$\left\{\begin{array}{l}\lambda_{1}=1, \lambda_{2}=0 \\ \beta_{1}=1, \beta_{2}=0.5\end{array}\right.$

Three algorithms are selected for the simulation comparing targets, including the adaptive space particle swarm optimization (RPSOAS) [12], combined differential evolution algorithm (CDE) [13] and parameter adaptive differential evolution algorithm (C-MC) [14], and running the RFID network optimization processes of these three algorithms for 50 times to obtain the average value, and the optimal and average values of 
RFID network optimization are shown in Table 2, and 3, and the optimal network identification distribution results obtained by means of comparing algorithms are shown in Figure 3. The evolution process convergence curve is as shown in Figure 4.

Table 2. Optimal Identification Results

\begin{tabular}{ccccc}
\hline & GTHS & CDE & RPSO AS & C-MC \\
\hline Coverage rate & 72.1356 & 85.3415 & 90.9584 & 83.9128 \\
load balance & 0.0123 & 0.0239 & 0.8065 & 0.0553 \\
network cost & 0.15 & 0.32 & 0.41 & 0.36 \\
target value & 5.2327 & 8.5368 & 9.1271 & 7.4357 \\
\hline
\end{tabular}

Table 3. Average Recognition Results

\begin{tabular}{ccccc}
\hline & GTHS & CDE & RPSO AS & C-MC \\
\hline Coverage rate & 69.3214 & 81.1798 & 86.1918 & 80.0345 \\
load balance & 0.0021 & 0.0223 & 0.299 & 0.0274 \\
network cost & 0.127 & 0.331 & 0.323 & 0.226 \\
target value & 4.9675 & 7.8935 & 8.0679 & 72286 \\
\hline
\end{tabular}

In the simulation results if Figure 3 (a), (d), the RFID network optimal identification conditions of four comparison algorithms, which are CTHS, RPSOAS, CDE and C-MC, are presented, and the black spots "." and surrounding small cireles are RFID network electronic tags and their uncertainty regions The bìg circles in simulation results represent the detection area of corresponding readers, and "*" represents the readers in RFID network. Table 2, and 3, the average optimization values of the four comparison algorithms which are GTHS, RPSOAS, CDE and C-MC for RFID network target function. Table 2, shows the optinal evolution results, from which it can be seen that in the comparison of optimization results of optimal adaptive values, GTHS algorithm can achieve better optimization result than those of the other three algorithms, while C-MC algorithm ranks the second place, achieving slightly better results than those of RPS0AS and CDE algorithms. Table 3, shows the optimization data of average adaptive values of the above-mentioned four algorithms, and the average value operation results in Table 3 are similar to those in Table 2, with the average value results of GTHS algorithm being better than those of the othey three algorithms, and C-MC algorithm ranking the second place, being slightly better than RPSOAS and CDE algorithms. The above experimental data directly proves the feasibility and effectiveness of GTHS algorithm in IOT RFID network identification

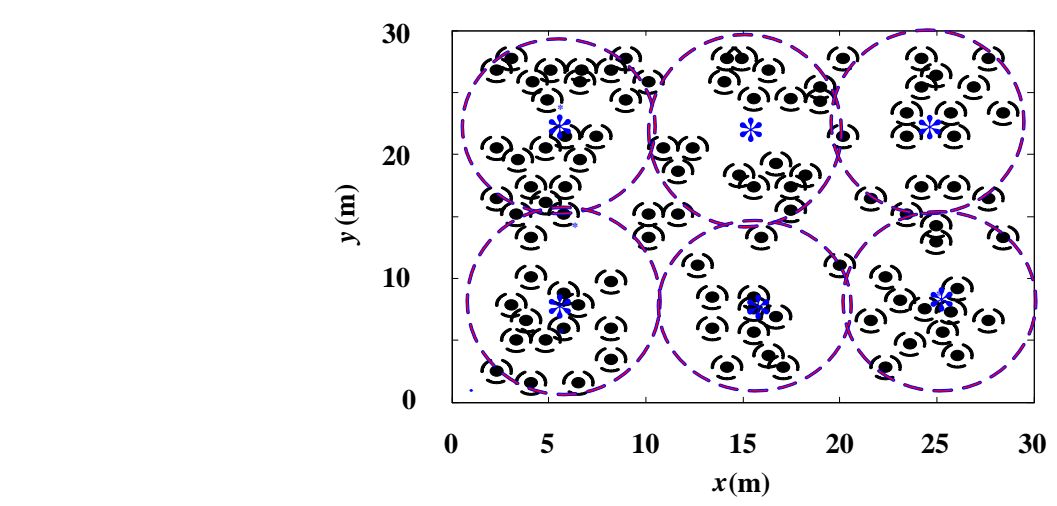

(a) Optimal identification results for GTHS algorithm 


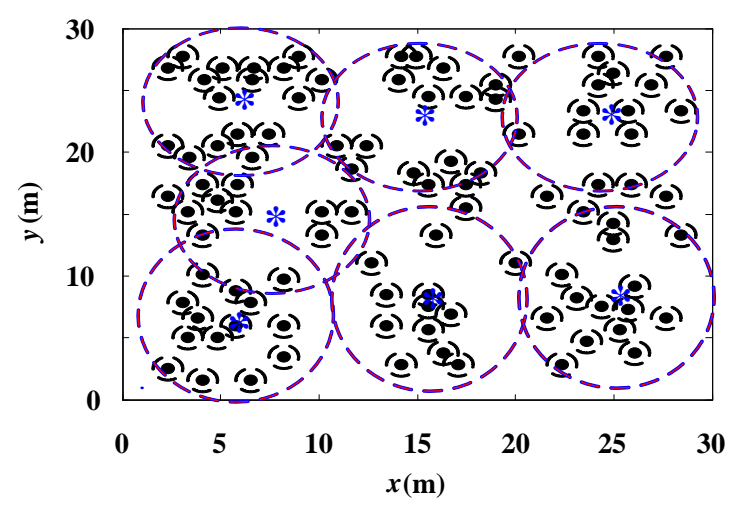

(b) Optimal identification results for CDE algorithm

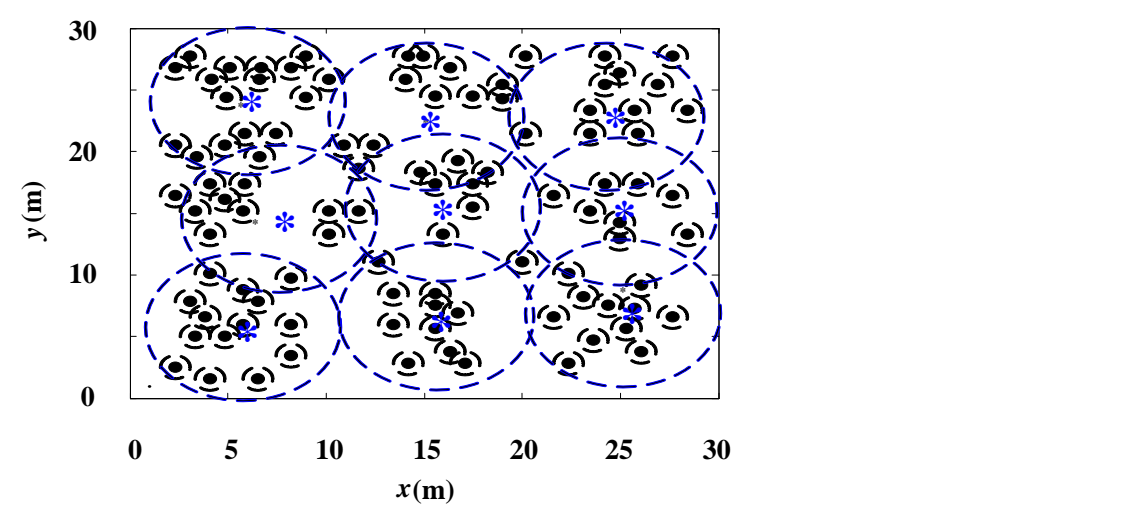

(c) Optimal identification results for RPSOAS algorithm

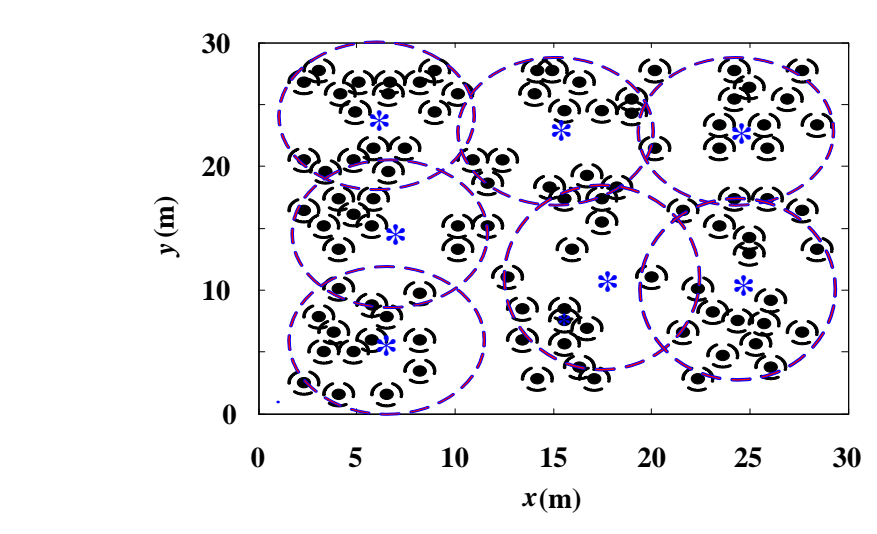

(d) Optimal identification results for C-MC algorithm

Figure 3. Optimal Identification Results

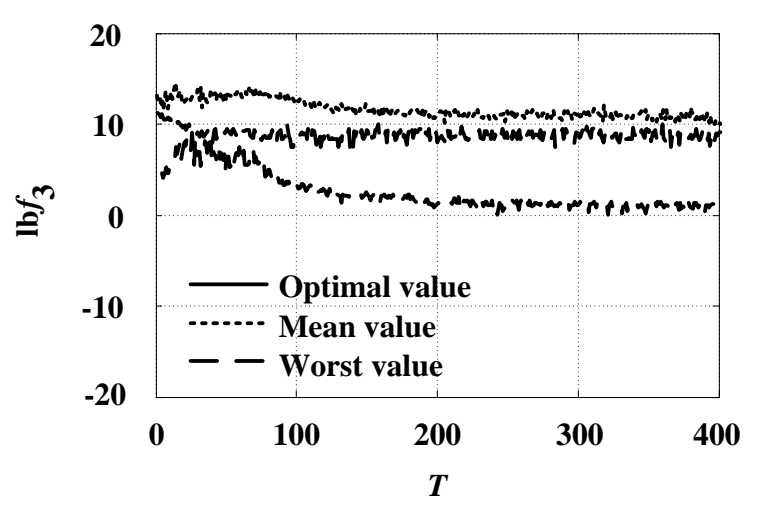




\section{(a) Coverage convergence curve}

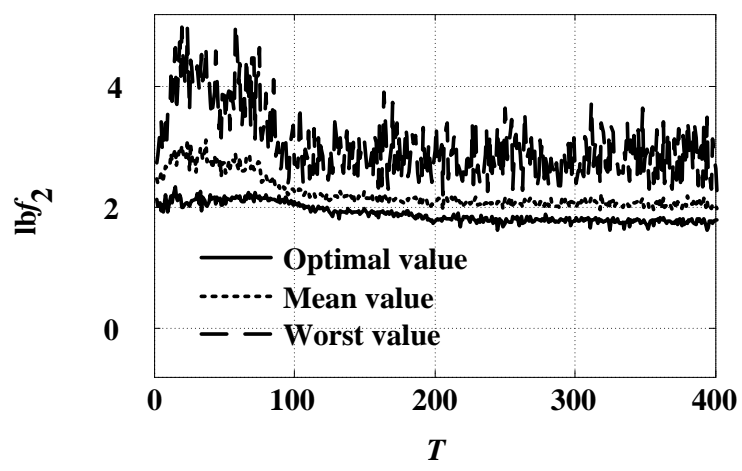

(b) Network cost convergence curve

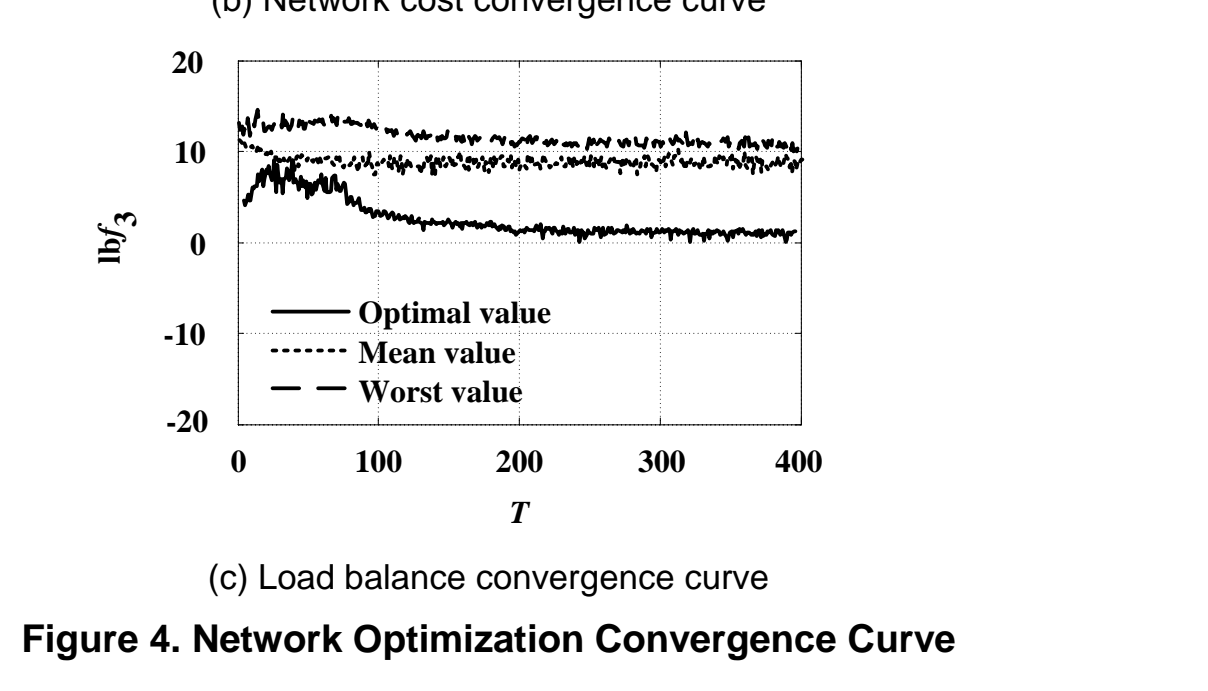

Figure 4, shows the optimal, worst and average convergence curves of target adaptive values when GTHS algonthm and adaptive target function are adopted, and in order to present the data differences, the binary logarithm which is commonly used in signal processing is used for data amplification. It can be seen from simulations curves that although the orders of magnitude of three targets' adaptive values are different, GTHS algorithm can aehieve synchronous optimization of the three network index after adopting the weighting adaptive for refinement, and meanwhile the phenomenon in Reference [8] that index with larger orders of magnitude merging index with smaller orders of magnitude can be avoided, so that the problem of local extreme points can be prevented.

Table 5, shows the load balancing and adaptive value deviation, from which it can be seen that, under the precondition of different network scales and tag distribution forms, CDE a gorithm has better performance in load balancing and adaptive value than PRSOAS and C-MC algorithms, and each of the later two algorithms has advantage and disadvantage in load balancing and adaptive value. Compared with the above three algorithms, GTHS algorithm always has the optimal load balancing and adaptive value, which proves the robustness of GTHS algorithm on network parameters.

Table 5. Load Balance and Fitness Deviation

\begin{tabular}{ccccccc}
\hline $\begin{array}{c}\text { Network } \\
\text { Scale }\end{array}$ & $\begin{array}{c}\text { Tag } \\
\text { distribution }\end{array}$ & Performance index & GTHS & CDE & RPSOAS & C-MC \\
\hline $30 \times 30 \mathrm{~m}$ & Normal & Load balance & 0.0278 & 0.0371 & 0.0417 & 0.3202 \\
$100 \mathrm{tags}$ & & Adaptive value & 7.2347 & 7.6164 & 7.8598 & 8.0525 \\
$50 \times 50 \mathrm{~m}$ & Normal & Load balance & 0.0312 & 0.0386 & 0.0624 & 0.3501 \\
\hline
\end{tabular}




\begin{tabular}{ccccccc}
\hline $\begin{array}{c}\text { Network } \\
\text { Scale }\end{array}$ & $\begin{array}{c}\text { Tag } \\
\text { distribution }\end{array}$ & Performance index & GTHS & CDE & RPSOAS & C-MC \\
\hline 250 tags & & Adaptive value & 7.2156 & 7.5601 & 7.753 & 7.8085 \\
& \multirow{2}{*}{ Random } & Load balance & 0.0326 & 0.0394 & 0.0838 & 0.3504 \\
& & Adaptive value & 7.1682 & 7.4611 & 7.6617 & 7.8183 \\
& \multirow{2}{*}{ Normal } & Load balance & 0.0326 & 0.0384 & 0.0511 & 0.3437 \\
$100 \times 100 \mathrm{~m}$ & & Adaptive value & 7.2863 & 7.5402 & 7.6073 & 7.7857 \\
1000 tags & \multirow{2}{*}{ Random } & Load balance & 0.0317 & 0.0352 & 0.0795 & 0.3451 \\
& & Adaptive value & 7.0563 & 7.6296 & 7.6812 & 7.8194 \\
\hline
\end{tabular}

\section{Conclusion}

In order to further improve the accuracy of IOT RFID network optimization, this paper puts forward the innovative design from two aspects: One is to further improve the optimization algorithm performance, which is based on harmony search algorithm design control parameters and introduce Gaussian traverse distribution function for refinement; the other is improvement of RFID network optimization model, which/designs adaptive weighting form target function to realize synchronous evolution of network index, effectively avoiding being trapped in extreme points. The simulation results show that the GTHS algorithm optimization result of adaptive veighting target function is better than those of algorithms in comparing references. The research on radio frequency network optimization of large-scale IOT RFID has very importanteconomic significance; the above-mentioned algorithms also include the researches on this field, but lack of verification under practical environment and this will be the focus of next step researches.

\section{Acknowledgements}

Tangshan city Science and Technology plan project, under Grant No. 15130204a.

\section{References}

[1] Z. Lv, A. Halawan, S. Feng, H. Ci and S. U. Rehman, "Multimodal Hand and Foot Gesture Interaction for Handheld Devices", ACM Transactions on Multimedia Computing, Communications, and Applications (TOMM), vol (1), no. 1, Article 10, (2014) October, pp. 19.

[2] Y. Lin, J. Yang, Z. Lv, W Wei and H. Song, "A Self-Assessment Stereo Capture Model Applicable to the Internet of Things", Sensors, (2015).

[3] W. Ou, Z. Lv and Z, Xje, "Spatially Regularized Latent topic Model for Simultaneous object discovery and segmentation", The 2015 IEEE International Conference on Systems, Man, and Cybernetics, (2015).

[4] Y. Wang, Y.Su and G. Agrawal, "A Novel Approach for Approximate Aggregations Over Arrays", In Proceedings of the 27th international conference on scientific and statistical database management, ACM, (2015).

[5] Z. Lv, A. Halawani, S. Feng, S. U. Rehman and H. Li, "Touch-less Interactive Augmented Reality Game on Vision Based Wearable Device", Personal and Ubiquitous Computing, (2015).

[6] Yang, S. He, Y. Lin and Z. Lv, "Multimedia cloud transmission and storage system based on internet f things", Multimedia Tools and Applications, (2016).

[7] D. Jiang, X. Ying, Y. Han and Z. Lv, "Collaborative Multi-hop Routing in Cognitive Wireless Networks", Wireless Personal Communications, (2015).

[8] W. Wang, Z. Lu, X. Li, W. Xu, B. Zhang and X. Zhang, "Virtual Reality Based GIS Analysis Platform", 22th International Conference on Neural Information Processing, Istanbul, Turkey, ICONIP, (2015).

[9] Z. Lv, C. Esteve, J. Chirivella and P. Gagliardo, "Clinical Feedback and Technology Selection of Game Based Dysphonic Rehabilitation Tool”, 2015 9th International Conference on Pervasive Computing Technologies for Healthcare (PervasiveHealth2015), IEEE, (2015).

[10] J. Yang, "A Low-Power and Portable Biomedical Device for Respiratory Monitoring with a Stable Power Source", Sensors, vol. 15, no. 8, (2015), pp. 19618-19632.

[11] Z. Chen, W. Huang and Z. Lv, "Towards a face recognition method based on uncorrelated discriminant sparse preserving projection", Multimedia Tools and Applications, (2015), pp. 1-15. 
[12] X. Li, "XEarth: A 3D GIS Platform for managing massive city information", Computational Intelligence and Virtual Environments for Measurement Systems and Applications (CIVEMSA), 2015 IEEE International Conference on. IEEE, (2015).

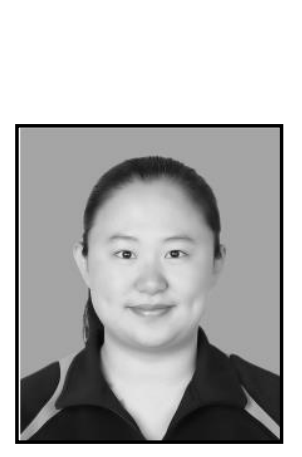

\begin{abstract}
Authors
Zhang Hongbo, received her M.Eng. degree in software engineering from Tongji University in Shanghai, China. She is currently a lecturer in the Department of Computer Science at Tangshan Normal University. Her research interest is mainly in the area of Computer Software, the Internet of things technology. She has published several research papers in scholarly journals in the above research areas.
\end{abstract}

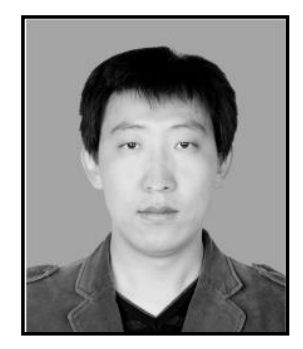

Gao Wei, received his M.Eng. degree in software engineering from Tongji University in Shanghai, China. He is currently lecturer in the Department of Computer Science at Tangshan Normal University. His research interest is mainy in the area of Computer Software, the Internet of things technology. He has published several research papers in scholarly journals in the above research areas.

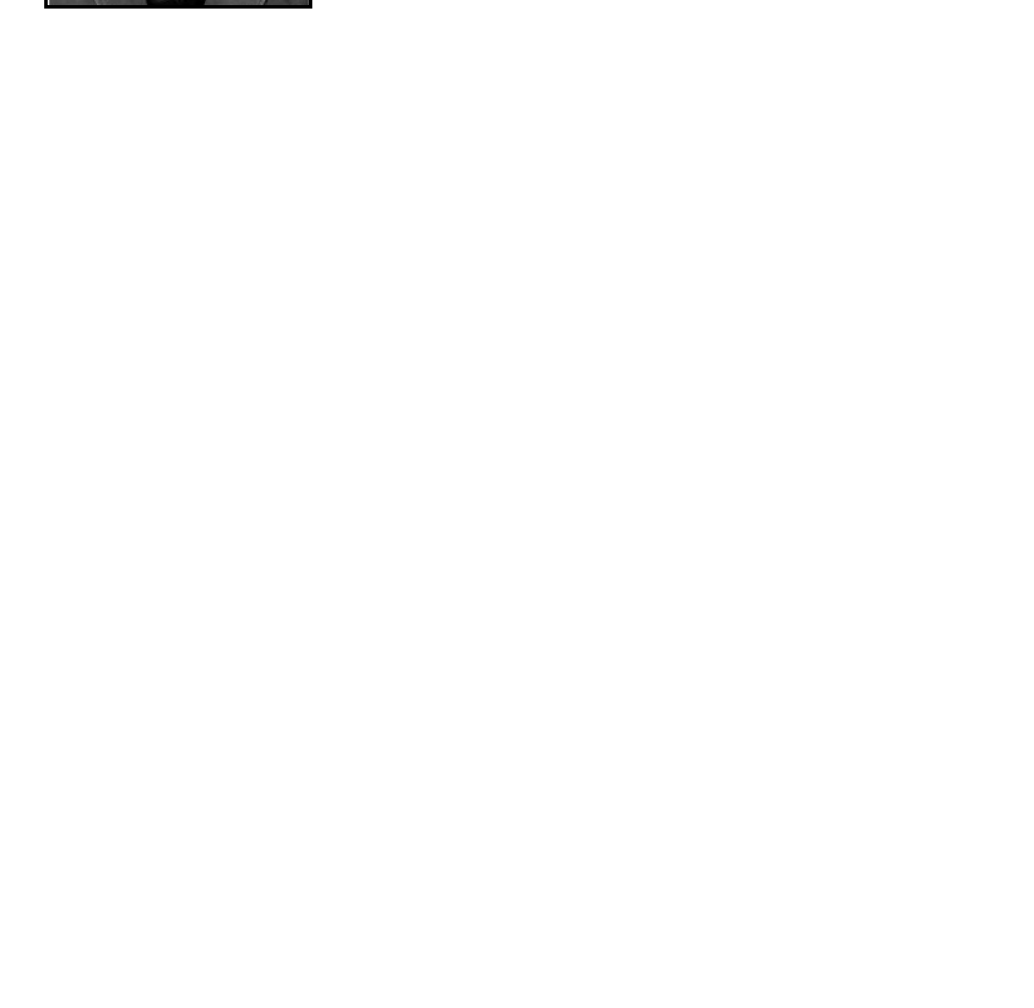


International Journal of Smart Home

Vol. 10, No. 11, (2016)

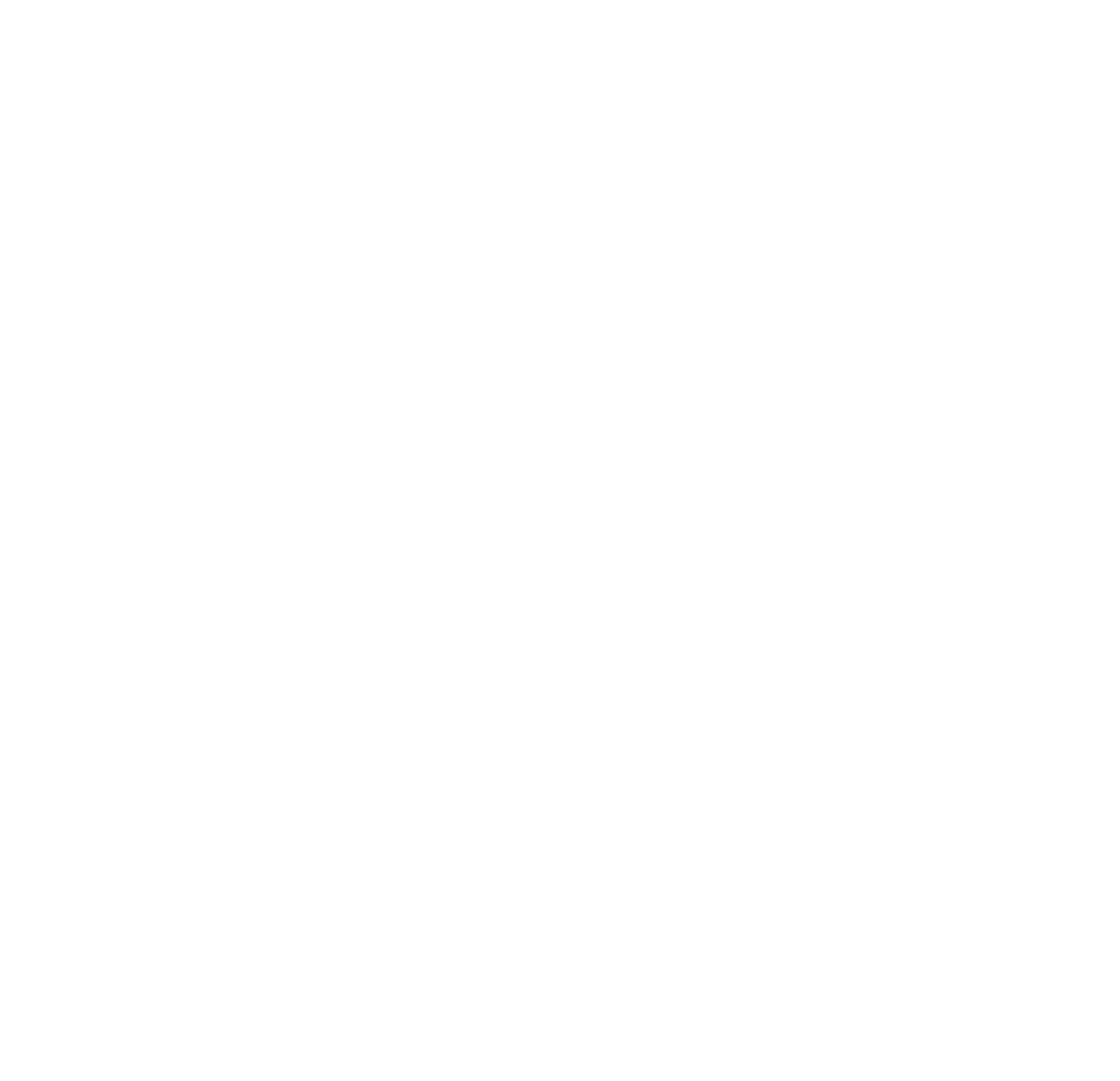

